\title{
ON THE EXTINCTION OF A CLASS \\ OF POPULATION-SIZE-DEPENDENT BISEXUAL BRANCHING PROCESSES
}

\author{
YONGSHENG XING * AND \\ YONGJIN WANG, ${ }^{* *}$ Nankai University
}

\begin{abstract}
In this paper, we study a class of bisexual Galton-Watson branching processes in which the law of offspring distribution is dependent on the population size. Under a suitable condition on the offspring distribution, we prove that the limit of mean growth-rate per mating unit exists. Based on this limit, we give a criterion to identify whether the process admits ultimate extinction with probability one.

Keywords: Bisexual Galton-Watson branching process; population-size-dependent branching process; extinction probability

2000 Mathematics Subject Classification: Primary 60J80
\end{abstract}

\section{Introduction of bisexual Galton-Watson branching processes}

The bisexual Galton-Watson process was first introduced by Daley (1968a) as a two-type branching model $\left\{\left(F_{n}, M_{n}\right), n=1,2, \ldots\right\}$, which can be described in the following way.

Let $F_{n}$ and $M_{n}$ respectively denote the number of females and males in the $n$th generation, and let $L(\cdot, \cdot)$ be a mating function that describes their mating rule. We denote by $Z_{n}$ the number of mating units in the $n$th generation. Moreover, $\xi_{n, j}$ and $\eta_{n, j}$ are the numbers of females and males produced by the $j$ th mating unit in the $n$th generation, respectively. To formulate the model, we begin with $Z_{0} \in \mathbb{N}^{+}$(the set of all nonnegative integers) and inductively define

$$
\begin{aligned}
\left(F_{n+1}, M_{n+1}\right) & :=\sum_{j=1}^{Z_{n}}\left(\xi_{n, j}, \eta_{n, j}\right), \quad n=0,1,2, \ldots, \\
Z_{n+1} & :=L\left(F_{n+1}, M_{n+1}\right), \quad n=0,1,2, \ldots
\end{aligned}
$$

(Here we use the convention that empty sums equal $(0,0)$ ).

We make the usual assumptions on the model, as follows. The $\left(\xi_{n, j}, \eta_{n, j}\right)$, where $n=$ $0,1, \ldots$ and $j=1,2, \ldots$, are independent, identically distributed, bivariate random variables taking values in $\mathbb{N}^{+} \times \mathbb{N}^{+}$, and the mating function $L: \mathbb{R}^{+} \times \mathbb{R}^{+} \rightarrow \mathbb{R}^{+}$is nondecreasing in each variable and satisfies the inequalities

$$
\begin{aligned}
L(x, y) & \leq x y, \\
L\left(\sum_{i=1}^{n} x_{i}, \sum_{i=1}^{n} y_{i}\right) & \geq \sum_{i=1}^{n} L\left(x_{i}, y_{i}\right)
\end{aligned}
$$

Received 29 September 2003; revision received 8 March 2004.

* Postal address: School of Mathematical Sciences and LPMC, Nankai University, Tianjin 300071, P. R. China.

Supported by the Natural Science Foundation of China (grant no. 10131040).

** Email address: yjwang@nankai.edu.cn 
for any $n \geq 2$ and any $(x, y),\left(x_{i}, y_{i}\right) \in \mathbb{R}^{+} \times \mathbb{R}^{+}$. (The second inequality is known as the superadditive property.)

Bisexual branching processes have received much attention in the literature. The extinction problem has been studied by Daley (1968a), Hull (1982), (1984), Bruss (1984), Daley et al. (1986), and Alsmeyer and Rösler (1996). The main result, proved by Daley et al. (1986), is based on the concept of mean growth-rate per mating unit, i.e.

$$
r_{k}:=k^{-1} \mathrm{E}\left[Z_{n+1} \mid Z_{n}=k\right], \quad k=1,2 \ldots,
$$

which was introduced by Bruss (1984). Daley et al. (1986) proved that, for a superadditive branching process, the asymptotic growth-rate $r:=\lim _{k \rightarrow \infty} r_{k}$ exists, and that

$$
\mathrm{P}\left(Z_{n} \rightarrow 0, n \rightarrow \infty \mid Z_{0}=j\right)=1, \quad j=1,2, \ldots,
$$

if and only if, excluding trivial cases,

$$
r \leq 1
$$

Molina et al. (2002) suggested a bisexual Galton-Watson model with population-sizedependent mating. They obtained a necessary and sufficient condition for the process to become extinct with probability 1 .

In this paper, we are interested in the so-called population-size-dependent bisexual GaltonWatson processes (PSDBPs), i.e. the class of bisexual Galton-Watson processes whose offspring reproduction laws depend on the size of the population. The biological motivation for this model is that population size governs reproduction laws.

In section 2, the probabilistic model is described and basic concepts and necessary results are introduced. Then, in section 3, we give a criterion (see Theorem 3.1) to identify whether the process admits ultimate extinction with probability one.

\section{The probabilistic model}

We define a population-size-dependent bisexual Galton-Watson process via a two-type sequence $\left(F_{n}^{\star}, M_{n}^{\star}\right)_{n}$, as follows:

$$
\begin{aligned}
& Z_{0}^{\star}:=N, \\
&\left(F_{n+1}^{\star}, M_{n+1}^{\star}\right):=\sum_{j=1}^{Z_{n}^{\star}}\left(\xi_{n, j}^{\left(Z_{n}^{\star}\right)}, \eta_{n, j}^{\left(Z_{n}^{\star}\right)}\right), \quad n=0,1, \ldots, \\
& Z_{n+1}^{\star}:=L\left(F_{n+1}^{\star}, M_{n+1}^{\star}\right), \quad n=0,1, \ldots
\end{aligned}
$$

Here, the law of offspring distribution depends only on the size of the parental generation. We assume that, for every $k=1,2, \ldots$, the random variables $\left(\xi_{n, j}^{(k)}, \eta_{n, j}^{(k)}\right)$, where $n=0,1 \ldots$, $j=1,2, \ldots$, are independent and have the same distribution as $\left(\xi_{0,1}^{(k)}, \eta_{0,1}^{(k)}\right)$. As usual, the mating function $L(\cdot, \cdot)$ is assumed to be superadditive (see (1.1)).

Remark 2.1. It is not hard to check that $\left\{Z_{n}^{\star}, n \geq 0\right\}$ is a homogeneous Markov chain and that 0 is an absorbing state. However, $\left\{Z_{n}^{\star}, n \geq 0\right\}$ is not a stochastically monotone Markov chain in the sense of Daley (1968b).

Throughout this paper, we suppose that the sequence of offspring random variables $\left(\xi_{0,1}^{(k)}, \eta_{0,1}^{(k)}\right)_{k}$ satisfies the following condition. 
Condition 2.1. The sequence $\left(\xi_{0,1}^{(k)}, \eta_{0,1}^{(k)}\right)_{k}$ satisfies

$$
\mathrm{E} g\left(\xi_{0,1}^{(k+1)}, \eta_{0,1}^{(k+1)}\right) \leq \mathrm{E} g\left(\xi_{0,1}^{(k)}, \eta_{0,1}^{(k)}\right)
$$

for every bounded, componentwise-increasing function $g(\cdot, \cdot)$.

Remark 2.2. It follows from Kamae et al. (1977) that, simultaneously for all $k \geq 1$, there exist random variables $\left(\xi^{(k)}, \eta^{(k)}\right)^{\star}$ and $\left(\xi^{(k+1)}, \eta^{(k+1)}\right)^{\star}$, defined on the same probability space and having the same (respective) distributions as $\left(\xi_{0,1}^{(k)}, \eta_{0,1}^{(k)}\right)$ and $\left(\xi_{0,1}^{(k+1)}, \eta_{0,1}^{(k+1)}\right)$, such that

$$
\left(\xi^{(k)}, \eta^{(k)}\right)^{\star}=\left(\xi^{(k+1)}, \eta^{(k+1)}\right)^{\star}+\left(\xi^{(k, k+1)}, \eta^{(k, k+1)}\right), \quad k=0,1, \ldots,
$$

for nonnegative, integer-valued random variables $\left(\xi^{(k, k+1)}, \eta^{(k, k+1)}\right)$.

In the following, we will consider the random variables $\left(\xi^{(k)}, \eta^{(k)}\right)^{\star}$ given in this remark, instead of $\left(\xi_{0,1}^{(k)}, \eta_{0,1}^{(k)}\right)$. However, in an abuse of notation, we will write $\left(\xi^{(k)}, \eta^{(k)}\right)$ for $\left(\xi^{(k)}, \eta^{(k)}\right)^{\star}$.

With this, we can immediately make the following proposition.

Proposition 2.1. Under Condition 2.1,

1. the sequence $\left(\xi^{(k)}, \eta^{(k)}\right)_{k}$ converges almost surely to a pair of nonnegative, integer-valued random variables $(\xi, \eta)$; and

2. the sequence $\left(\mathrm{E} g\left(\xi^{(k)}, \eta^{(k)}\right)\right)_{k}$ is monotonic, nonincreasing, and converges to $\mathrm{E} g(\xi, \eta)$, where $g(\cdot, \cdot)$ is defined as in Condition 2.1.

So, letting $g(x, y)=x$ and $g(x, y)=y$ in turn, we have, respectively,

$$
\lim _{k \rightarrow \infty} \mathrm{E} \xi^{(k)}=\mathrm{E} \xi \quad \text { and } \quad \lim _{k \rightarrow \infty} \mathrm{E} \eta^{(k)}=\mathrm{E} \eta
$$

In order to obtain a sufficient condition for the process to become extinct, i.e. to have $Z_{n}^{\star}=0$ with probability 1 for some positive integer $n$, we must define some characteristic quantities.

Definition 2.1. We define ultimate extinction to be the event $Q:=\left\{Z_{n}^{\star} \rightarrow 0, n \rightarrow \infty\right\}$, and let

$$
q(j):=\mathrm{P}\left(Q \mid Z_{0}^{\star}=j\right), \quad j=1,2, \ldots
$$

(Actually, $Q=\bigcup_{n=1}^{\infty}\left\{Z_{n}^{\star}=0\right\}$.)

The following assertion originally comes from Molina et al. (2002), but the proof needs some modifications in our case.

Proposition 2.2. Let $\left\{Z_{n}^{\star}, n \geq 0\right\}$ be a PSDBP satisfying Condition 2.1, and suppose that the mating function satisfies $L(1,1)=1$. If

$$
\mathrm{P}\left(Z_{n+1}^{\star}=j \mid Z_{n}^{\star}=j\right)<1 \text { holds for all } j=1,2, \ldots,
$$

then

$$
\mathrm{P}\left(Z_{n}^{\star} \rightarrow 0\right)+\mathrm{P}\left(Z_{n}^{\star} \rightarrow \infty\right)=1 .
$$

Proof. It suffices to prove that, if $k \neq 0$, then $k$ is transient in the Markov chain $\left\{Z_{n}^{\star}, n \geq 0\right\}$.

Step 1. Suppose that, for any $k=1,2, \ldots$ and $j=0,1, \ldots$, the offspring distribution satisfies

$$
\mathrm{P}\left(\xi^{(k)}=0, \eta^{(k)}=j\right)=\mathrm{P}\left(\xi^{(k)}=j, \eta^{(k)}=0\right)=0 .
$$


This means that every mating unit has among its offspring at least one female and one male. Since $L(1,1)=1$, we have

$$
Z_{1}^{\star} \leq Z_{2}^{\star} \leq Z_{3}^{\star} \leq \cdots
$$

It follows that if

$$
f_{k k}^{\star}:=\mathrm{P}\left(Z_{n+m}^{\star}=k \text { for some } m \geq 1 \mid Z_{n}^{\star}=k\right),
$$

then

$$
f_{k k}^{\star}=\mathrm{P}\left(Z_{n+1}^{\star}=k \mid Z_{n}^{\star}=k\right)<1 .
$$

This implies that $k$ is transient.

Step 2. Suppose that there exists a $k \geq 1$ and a $j \geq 0$ such that either

$$
\mathrm{P}\left(\xi^{(k)}=0, \eta^{(k)}=j\right)>0 \quad \text { or } \quad \mathrm{P}\left(\xi^{(k)}=j, \eta^{(k)}=0\right)>0 .
$$

Then either

$$
\mathrm{P}\left(\xi^{(k)}=0\right)>0 \quad \text { or } \quad \mathrm{P}\left(\eta^{(k)}=0\right)>0 .
$$

Let

$$
N:=\inf \left\{k \geq 1: \mathrm{P}\left(\xi^{(k)}=0\right)>0 \text { or } \mathrm{P}\left(\eta^{(k)}=0\right)>0\right\} .
$$

It follows, from Remark 2.1, that either

$$
\mathrm{P}\left(\xi^{(N+m)}=0\right)>0 \quad \text { or } \quad \mathrm{P}\left(\eta^{(N+m)}=0\right)>0
$$

for all $m=0,1,2, \ldots$

Since $L(x, y) \leq x y$, we have $L(0, \cdot)=L(\cdot, 0)=0$ and, therefore, for $k \geq N$,

$$
\begin{aligned}
\mathrm{P}\left(Z_{n+1}^{\star}=0 \mid Z_{n}^{\star}=k\right) & =\mathrm{P}\left(L\left(\sum_{j=1}^{k}\left(\xi_{n, j}^{(k)}, \eta_{n, j}^{(k)}\right)\right)=0\right) \\
& \geq \mathrm{P}\left(\sum_{j=1}^{k} \xi_{n, j}^{(k)}=0 \text { or } \sum_{j=1}^{k} \eta_{n, j}^{(k)}=0\right) \\
& \geq \max \left\{\mathrm{P}\left(\sum_{j=1}^{k} \xi_{n, j}^{(k)}=0\right), \mathrm{P}\left(\sum_{j=1}^{k} \eta_{n, j}^{(k)}=0\right)\right\} \\
& =\max \left\{\mathrm{P}\left(\xi^{(k)}=0\right)^{k}, \mathrm{P}\left(\eta^{(k)}=0\right)^{k}\right\} \\
& >0 .
\end{aligned}
$$

Since 0 is an absorbing state, we deduce that

$$
f_{k k}^{\star} \leq 1-\mathrm{P}\left(Z_{n+1}^{\star}=0 \mid Z_{n}^{\star}=k\right)<1 .
$$

This implies that $k$ is transient.

For $k<N$, by the definition of $N$, we have

$$
\mathrm{P}\left(\xi^{(k)}=0\right)=\mathrm{P}\left(\eta^{(k)}=0\right)=0 .
$$

So, $\mathrm{P}\left(\xi^{(k)}=0, \eta^{(k)}=j\right)=\mathrm{P}\left(\xi^{(k)}=j, \eta^{(k)}=0\right)=0$ for all $j=0,1, \ldots$

We claim that

$$
P\left(Z_{n}^{\star} \geq N \text { for some } n \geq 1 \mid Z_{0}^{\star}=k\right)>0 .
$$


Otherwise

$$
\mathrm{P}\left(Z_{n}^{\star} \geq N \mid Z_{0}^{\star}=k\right)=0 \quad \text { for every } n \geq 1,
$$

which implies that

$$
Z_{0}^{\star} \leq Z_{1}^{\star} \leq Z_{2}^{\star} \leq \cdots<N
$$

contradicting (2.3). Hence, the states no smaller than $N$ are accessible from $k$, and $k$ is transient.

In summary, $k \neq 0$ is transient for the Markov chain $\left\{Z_{n}^{\star}, n \geq 0\right\}$, and the assertion of the proposition follows.

\section{Extinction probability under Condition 2.1}

In this section, we will study the extinction problem of a PSDBP under Condition 2.1. By developing some techniques similar to those of Daley et al. (1986) and Molina et al. (2002), we obtain a criterion analogous to theirs for superadditive branching processes.

For our purposes, we introduce the 'mean growth-rates per mating unit' for a PSDBP.

Definition 3.1. Let $\left\{Z_{n}^{\star}, n \geq 0\right\}$ be a PSDBP. For every positive integer $k$, we define the mean growth-rate per mating unit as

$$
r_{k}^{\star}:=\frac{1}{k} \mathrm{E}\left[Z_{n+1}^{\star} \mid Z_{n}^{\star}=k\right]=\frac{1}{k} \mathrm{E} L\left(\sum_{j=1}^{k}\left(\xi_{n, j}^{(k)}, \eta_{n, j}^{(k)}\right)\right) .
$$

Proposition 3.1. Assume that the PSDBP $\left\{Z_{n}^{\star}, n \geq 0\right\}$ satisfies Condition 2.1. Then the limit $r^{\star}:=\lim _{k \rightarrow \infty} r_{k}^{\star}$ exists.

Remark 3.1. Proposition 3.1 plays a key role in the derivation of the extinction probability. However, for the PSDBP, the function $\psi$ defined by $\psi(j):=j r_{j}^{\star}$ is not superadditive, so the well-known method described in Daley et al. (1986) does not work in our case.

Proof of Proposition 3.1. The proof is done in two steps.

Step 1. For every $m \geq 1$, we define a bisexual Galton-Watson process $\left\{Z_{n}^{(m)}, n \geq 0\right\}$ as follows:

$$
\begin{aligned}
& Z_{0}^{(m)}:=N \\
& \left(F_{n+1}^{(m)}, M_{n+1}^{(m)}\right):=\sum_{j=1}^{Z_{n}^{(m)}}\left(\xi_{n, j}^{(m)}, \eta_{n, j}^{(m)}\right), \quad n=0,1,2, \ldots, \\
& Z_{n+1}^{(m)}:=L\left(F_{n+1}^{(m)}, M_{n+1}^{(m)}\right), \quad n=0,1,2, \ldots,
\end{aligned}
$$

where mating units of each generation produce offspring independently, but with the same offspring probability distribution as $\left(\xi^{(m)}, \eta^{(m)}\right)$, i.e.

$$
\mathrm{E} \xi^{(m)}=\mathrm{E} \xi_{n, j}^{(m)} \quad \text { and } \quad \mathrm{E} \eta^{(m)}=\mathrm{E} \eta_{n, j}^{(m)},
$$

for all $n=0,1, \ldots, j=1,2, \ldots$ By (2.2), we have

$$
\lim _{m \rightarrow \infty} \mathrm{E} \xi^{(m)}=\mathrm{E} \xi \text { and } \quad \lim _{m \rightarrow \infty} \mathrm{E} \eta^{(m)}=\mathrm{E} \eta
$$


Let

$$
r_{k}^{(m)}:=\frac{1}{k} \mathrm{E}\left[Z_{n+1}^{(m)} \mid Z_{n}^{(m)}=k\right]=\frac{1}{k} \mathrm{E} L\left(\sum_{j=1}^{k}\left(\xi_{n, j}^{(m)}, \eta_{n, j}^{(m)}\right)\right) .
$$

Then, by Theorem 1 of Daley et al. (1986), the limit $r^{(m)}:=\lim _{k \rightarrow \infty} r_{k}^{(m)}$ exists, with $r^{(m)}=$ $\sup _{k>0} r_{k}^{(m)}$. Furthermore, by Theorem 3.2 of Molina et al. (2002),

$$
r^{(m)}=r\left(\mathrm{E} \xi^{(m)}, \mathrm{E} \eta^{(m)}\right)
$$

where the function $r(x, y)$ is continuous for every nonnegative-valued $(x, y)$ - see Proposition 3.2 of Molina et al. (2002). So, by (3.1), the limit $r:=\lim _{m \rightarrow \infty} r^{(m)}$ exists, and

$$
r=r(\mathrm{E} \xi, \mathrm{E} \eta)
$$

Next, we consider a bisexual Galton-Watson process $\left\{\tilde{Z}_{n}, n \geq 0\right\}$ with the same offspring probability distribution as that of $(\xi, \eta)$, i.e.

$$
\begin{aligned}
\tilde{Z}_{0} & :=N, \\
\left(\tilde{F}_{n+1}, \tilde{M}_{n+1}\right) & :=\sum_{j=1}^{\tilde{Z}_{n}}\left(\xi_{n, j}, \eta_{n, j}\right), \\
\tilde{Z}_{n+1} & :=L\left(\tilde{F}_{n+1}, \tilde{M}_{n+1}\right) .
\end{aligned}
$$

(It is worth mentioning that $\left\{\tilde{Z}_{n}, n \geq 0\right\}$ is a stochastically monotone Markov chain.)

By Theorem 1 of Daley et al. (1986) and Theorem 3.2 of Molina et al. (2002), $\tilde{r}:=$ $\lim _{k \rightarrow \infty} k^{-1} \mathrm{E}\left[\tilde{Z}_{n+1} \mid \tilde{Z}_{n}=k\right]$ exists, with

$$
\tilde{r}=\sup _{k \geq 1} \frac{1}{k} \mathrm{E} L\left(\sum_{j=1}^{k}\left(\xi_{n, j}, \eta_{n, j}\right)\right)=r(\mathrm{E} \xi, \mathrm{E} \eta) .
$$

It follows, from (3.2) and (3.3), that

$$
r=\tilde{r}=r(\mathrm{E} \xi, \mathrm{E} \eta)
$$

Step 2. By Proposition 2.1(2), we have

$$
\frac{1}{k} \mathrm{E} L\left(\sum_{j=1}^{k}\left(\xi_{n, j}^{(k)}, \eta_{n, j}^{(k)}\right)\right) \geq \frac{1}{k} \mathrm{E} L\left(\sum_{j=1}^{k}\left(\xi_{n, j}, \eta_{n, j}\right)\right), \quad k \geq 1,
$$

and so

$$
\liminf _{k \rightarrow \infty} \frac{1}{k} \mathrm{E} L\left(\sum_{j=1}^{k}\left(\xi_{n, j}^{(k)}, \eta_{n, j}^{(k)}\right)\right) \geq \tilde{r} .
$$

For every $m \geq 1$, by applying Proposition 2.1(2), we find that

$$
\limsup _{k \rightarrow \infty} \frac{1}{k} \mathrm{E} L\left(\sum_{j=1}^{k}\left(\xi_{n, j}^{(k)}, \eta_{n, j}^{(k)}\right)\right) \leq \lim _{k \rightarrow \infty} \frac{1}{k} \mathrm{E} L\left(\sum_{j=1}^{k}\left(\xi_{n, j}^{(m)}, \eta_{n, j}^{(m)}\right)\right) .
$$


This implies that

$$
\limsup _{k \rightarrow \infty} \frac{1}{k} \mathrm{E} L\left(\sum_{j=1}^{k}\left(\xi_{n, j}^{(k)}, \eta_{n, j}^{(k)}\right)\right) \leq r^{(m)}
$$

and so

$$
\limsup _{k \rightarrow \infty} \frac{1}{k} \mathrm{E} L\left(\sum_{j=1}^{k}\left(\xi_{n, j}^{(k)}, \eta_{n, j}^{(k)}\right)\right) \leq r .
$$

Combining (3.4), (3.5), and (3.6), we deduce that $r^{\star}=\lim _{k \rightarrow \infty} k^{-1} \mathrm{E} L\left(\sum_{j=1}^{k}\left(\xi_{n, j}^{(k)}, \eta_{n, j}^{(k)}\right)\right)$ exists, with

$$
r^{\star}=r=\tilde{r}
$$

Theorem 3.1. For the PSDBP $\left\{Z_{n}^{\star}, n \geq 0\right\}$ satisfying Condition 2.1, the following assertions hold.

1. If $r^{\star}<1$ then $q(j)=1$ for $j=1,2, \ldots$

2. If $r^{\star}>1$ then $q(j)<1$ for $j=1,2, \ldots$.

Proof. 1. If $r^{\star}<1$, we have $r=r^{\star}<1$, by (3.7), recalling that $r=\lim _{m \rightarrow \infty} r^{(m)}$. Therefore, there exist at most finitely many $m$ s such that $r^{(m)} \geq 1$, and we can thus suppose that there exists a positive integer $k$ with $r^{(l+k)}<1$ for all $l>0$. Let $\alpha:=\max \left\{1, r^{(1)}, r^{(2)}, \ldots, r^{(k)}\right\}$. Since $r^{(m)}=\sup _{k>0} r_{k}^{(m)}$, we have

$$
\begin{aligned}
\mathrm{E} Z_{n+1}^{\star} & =\sum_{m=0}^{\infty} \mathrm{P}\left(Z_{n}^{\star}=m\right) \mathrm{E}\left[Z_{n+1}^{\star} \mid Z_{n}^{\star}=m\right] \\
& =\sum_{m=0}^{\infty} \mathrm{P}\left(Z_{n}^{\star}=m\right) \mathrm{E} L\left(\sum_{j=1}^{m}\left(\xi_{n, j}^{(m)}, \eta_{n, j}^{(m)}\right)\right) \\
& =\sum_{m=1}^{\infty} \mathrm{P}\left(Z_{n}^{\star}=m\right) m r_{m}^{(m)} \quad\left(\text { by the definition of } r_{k}^{(m)}\right) \\
& =\sum_{m=1}^{k} \mathrm{P}\left(Z_{n}^{\star}=m\right) m r_{m}^{(m)}+\sum_{m=k+1}^{\infty} \mathrm{P}\left(Z_{n}^{\star}=m\right) m r_{m}^{(m)} \\
& \leq \alpha \sum_{m=1}^{k} m \mathrm{P}\left(Z_{n}^{\star}=m\right)+\sum_{m=k+1}^{\infty} m \mathrm{P}\left(Z_{n}^{\star}=m\right) \\
= & \mathrm{E} Z_{n}^{\star}+(\alpha-1) \sum_{m=1}^{k} m \mathrm{P}\left(Z_{n}^{\star}=m\right)
\end{aligned}
$$

This implies that

$$
\mathrm{E} Z_{n+1}^{\star} \leq \mathrm{E} Z_{n}^{\star}+(\alpha-1) \sum_{m=1}^{k} m \mathrm{P}\left(Z_{n}^{\star}=m\right), \quad n \geq 0 .
$$

Iterating this inequality, we find that

$$
\mathrm{E} Z_{n}^{\star} \leq \mathrm{E} Z_{0}^{\star}+(\alpha-1) \sum_{m=1}^{k} m \sum_{j=0}^{n-1} \mathrm{P}\left(Z_{j}^{\star}=m\right),
$$


recalling that, if $m \neq 0, m$ is transient for $\left\{Z_{n}^{\star}, n \geq 0\right\}$. Furthermore, if we define $\lambda_{m}:=$ $\sum_{j=0}^{\infty} \mathrm{P}\left(Z_{j}^{\star}=m\right)<\infty$, we have

$$
\mathrm{E} Z_{n}^{\star} \leq \mathrm{E} Z_{0}^{\star}+(\alpha-1) \sum_{m=1}^{k} m \lambda_{m}<\infty \quad \text { for all } n .
$$

This implies that

$$
\mathrm{P}\left(Z_{n}^{\star} \rightarrow \infty, n \rightarrow \infty\right)=0
$$

i.e. $q(j)=1$.

2. If $r^{\star}>1$ then $\tilde{r}=r^{\star}>1$, by (3.7). Recall that $\left\{Z_{n}^{\star}, n \geq 0\right\}$ and $\left\{\tilde{Z}_{n}, n \geq 0\right\}$ have, respectively, the offspring random variables $\left(\xi_{n, j}^{(m)}, \eta_{n, j}^{(m)}\right)$ and $\left(\xi_{n, j}, \eta_{n, j}\right)$, where $m$ represents the present population size of $\left\{Z_{n}^{\star}, n \geq 0\right\}$.

By Proposition 2.1(2),

$$
\mathrm{E} g\left(\xi_{n, j}^{(m)}, \eta_{n, j}^{(m)}\right) \geq \mathrm{E} g\left(\xi_{n, j}, \eta_{n, j}\right),
$$

where $g(\cdot, \cdot)$ is as defined in (2.1). So, from our Remark 2.1 and Theorem 1 of Daley (1968b), we have

$$
\mathrm{P}\left(Z_{n}^{\star} \rightarrow 0 \mid Z_{0}^{\star}=j\right) \leq \mathrm{P}\left(\tilde{Z}_{n} \rightarrow 0 \mid \tilde{Z}_{0}=j\right) .
$$

Since $\tilde{r}>1$, we see that $\mathrm{P}\left(\tilde{Z}_{n} \rightarrow 0 \mid \tilde{Z}_{0}=j\right)<1$. Therefore $\mathrm{P}\left(Z_{n}^{\star} \rightarrow 0 \mid Z_{0}^{\star}=j\right)<1$, i.e. $q(j)<1$.

In the following, we give an example to illustrate that the extinction property is not certain when $r^{\star}=1$. This example demonstrates (see Proposition 3.2, below) that the extinction argument depends heavily on the convergence rate of $r^{(m)} \rightarrow 1$.

For the PSDBP $\left\{Z_{n}^{\star}, n \geq 0\right\}$ with promiscuous mating, i.e. where the mating function satisfies $L(x, y)=x \min \{1, y\}$, we have, by the definition of $\left\{Z_{n}^{(m)}, n \geq 0\right\}$,

$$
\begin{aligned}
r^{(m)} & =\lim _{k \rightarrow \infty} \frac{1}{k} \mathrm{E}\left[Z_{n+1}^{(m)} \mid Z_{n}^{(m)}=k\right] \\
& =\lim _{k \rightarrow \infty} \frac{1}{k} \mathrm{E} L\left(\sum_{j=1}^{k}\left(\xi_{n, j}^{(m)}, \eta_{n, j}^{(m)}\right)\right) \\
& =\lim _{k \rightarrow \infty} \frac{1}{k} \mathrm{E}\left[\left(\sum_{j=1}^{k} \xi_{n, j}^{(m)}\right) \min \left\{1, \sum_{j=1}^{k} \eta_{n, j}^{(m)}\right\}\right] \\
& =\lim _{k \rightarrow \infty} \mathrm{E} \xi_{n, j}^{(m)} \mathrm{P}\left(\sum_{j=1}^{k} \eta_{n, j}^{(m)}>0\right) \\
& =\lim _{k \rightarrow \infty} \mathrm{E} \xi_{n, j}^{(m)}\left[1-\mathrm{P}\left(\eta_{n, j}^{(m)}=0\right)^{k}\right] \\
& =\mathrm{E} \xi_{n, j}^{(m)} \\
& =\mathrm{E} \xi^{(m)} .
\end{aligned}
$$

Let $\left\{F_{n}^{\star}, n \geq 0\right\}$ be an asexual Galton-Watson process defined by $F_{n+1}^{\star}:=\sum_{j=1}^{F_{n}^{\star}} \xi_{n, j}^{\left(F_{n}^{\star}\right)}$. This is actually a population-size-dependent Galton-Watson process (see Klebaner (1984)). Define 
$q^{\prime}:=\mathrm{P}\left(F_{n}^{\star} \rightarrow 0, n \rightarrow \infty\right)$ and let $\mu_{1}^{(m)}$ and $\sigma_{1}^{(m)}$ be, respectively, the mean and the variance of the offspring distribution, where $m$ is the population size. It is easy to see that $\mu_{1}^{(m)}=\mathrm{E} \xi^{(m)}=$ $r^{(m)}$. By letting $g(x, y)=x^{2}$ in Proposition 2.1(2), we see that $\sigma_{1}:=\lim _{m \rightarrow \infty} \sigma_{1}^{(m)}$ exists.

Let us assume that one further condition holds.

Condition 3.1. $\lim _{m \rightarrow \infty} m\left(r^{(m)}-1\right)=c$ for $0 \leq c<\infty$.

Then the conditions of Theorem 1.4 of Klebaner (1984) are satisfied, and we have the following proposition.

Proposition 3.2. Suppose that $c \neq \sigma_{1}$. Then,

(a) if $c>\sigma_{1}$ then $q^{\prime}<1$; and

(b) if $0<\sigma_{1}$ and lim $\sup _{m \rightarrow \infty} m^{\alpha}\left(r^{(m)}-1\right)<\infty, \alpha \geq 2$, then $q^{\prime}=1$.

The PSDBP $\left\{Z_{n}^{\star}, n \geq 0\right\}$ with promiscuous mating is a 'killed' Markov chain, which kills the process $\left\{F_{n}^{\star}, n \geq 0\right\}$ at state $j$ with probability $k(j)$, where $k(j)=r(j)^{j}$ and $r(j)=\mathrm{P}\left(\eta^{(j)}=\right.$ 0 ) for $j \in N$. Indeed, if $F_{0}^{\star}=j$, this process is killed if no males are produced, which happens with probability $k(j)$. It is thus easy to see that

$$
\mathrm{P}\left(F_{n}^{\star} \rightarrow 0, n \rightarrow \infty \mid F_{0}^{\star}=j\right) \leq \mathrm{P}\left(Z_{n}^{\star} \rightarrow 0, n \rightarrow \infty \mid Z_{0}^{\star}=j\right) .
$$

Theorem 3.2. For the PSDBP $\left\{Z_{n}^{\star}, n \geq 0\right\}$ with promiscuous mating satisfying Conditions 2.1 and 3.1, we have the following:

(a) $c>\sigma_{1}$ implies that $q(j)<1$; and

(b) $0<\sigma_{1}$ and $\lim \sup _{m \rightarrow \infty} m^{\alpha}\left(r^{(m)}-1\right)<\infty, \alpha \geq 2$, imply that $q(j)=1$.

Proof. (a) By the definition of $q(j)$,

$$
\begin{aligned}
q(j) & =1-\mathrm{P}\left(Z_{n}^{\star} \geq 1 \text { for all } n \geq 0 \mid Z_{0}^{\star}=j\right) \\
& =1-\sum_{i=1}^{\infty} \mathrm{P}\left(Z_{1}^{\star}=i \mid Z_{0}^{\star}=j\right) \mathrm{P}\left(Z_{n}^{\star} \geq 1 \text { for all } n \geq 1 \mid Z_{1}^{\star}=i\right) \\
& =1-\mathrm{E}_{j}\left[(1-k(j)) \mathrm{P}\left(Z_{n}^{\star} \geq 1 \mid Z_{1}^{\star}=F_{1}^{\star}\right)\right] \quad\left(\text { where } \mathrm{E}_{j}[\cdot]:=\mathrm{E}\left[\cdot \mid Z_{0}^{\star}=j\right]\right) \\
& =1-\mathrm{E}_{j} \prod_{n \geq 0}\left(1-k\left(F_{n}^{\star}\right)\right) .
\end{aligned}
$$

Let $Q^{\prime}:=\left\{F_{n}^{\star} \rightarrow \infty, n \rightarrow \infty\right\}$. By applying Proposition 3.2, we find that $\mathrm{P}\left(Q^{\prime}\right)>0$. Therefore, we need only show that $\prod_{n \geq 0}\left(1-k\left(F_{n}^{\star}\right)\right)>0$ on $Q^{\prime}$. Note that

$$
\begin{aligned}
\mathrm{E}_{j} \sum_{n \geq 0} k\left(F_{n}^{\star}\right) & =\mathrm{E}_{j} \sum_{n \geq 0} r\left(F_{n}^{\star}\right)^{F_{n}^{\star}} \\
& \leq \mathrm{E}_{j} \sum_{n \geq 0} r(1)^{F_{n}^{\star}} \\
& =\sum_{n \geq 0} \sum_{k=1}^{\infty} r(1)^{k} \mathrm{P}_{j}\left(F_{n}^{\star}=k\right) \quad\left(\text { where } \mathrm{P}_{j}(\cdot):=\mathrm{P}\left(\cdot \mid Z_{0}^{\star}=j\right)\right) \\
& =\sum_{k=1}^{\infty} r(1)^{k} \sum_{n \geq 0} \mathrm{P}_{j}\left(F_{n}^{\star}=k\right) .
\end{aligned}
$$


Let $G_{j k}:=\sum_{n \geq 0} \mathrm{P}_{j}\left(F_{n}^{\star}=k\right)$ be the Green function of the chain $\left\{F_{n}^{\star}, n \geq 0\right\}$. Then $G_{j k}<\infty$ for all $k \in N$, since $k$ is transient (see Klebaner (1984), p. 32). Thus, we obtain

$$
\mathrm{E}_{j} \sum_{n \geq 0} k\left(F_{n}^{\star}\right) \leq \sum_{k=1}^{\infty} r(1)^{k} G_{j k} .
$$

On the other hand, $\sum_{k=1}^{\infty} k^{-2} G_{j k}<\infty$ (see Klebaner (1984), p. 35), so we have

$$
\mathrm{E}_{j} \sum_{n \geq 0} k\left(F_{n}^{\star}\right)<\infty \quad \text { on } Q^{\prime} .
$$

This implies that $\prod_{n \geq 0}\left(1-k\left(F_{n}^{\star}\right)\right)>0$ on $Q^{\prime}$.

(b) By Proposition 3.2, we have

$$
\mathrm{P}\left(F_{n}^{\star} \rightarrow 0, n \rightarrow \infty \mid F_{0}^{\star}=j\right)=1 .
$$

So, by (3.8), we see that $q(j)=\mathrm{P}\left(Z_{n}^{\star} \rightarrow 0, n \rightarrow \infty \mid Z_{0}^{\star}=j\right)=1$. This completes the proof of Theorem 3.2 .

\section{Acknowledgement}

The authors are indebted to an anonymous referee for valuable comments and suggestions to improve an earlier version of the paper.

\section{References}

Alsmeyer, G. AND RöSLer, U. (1996). The bisexual Galton-Watson process with promiscuous mating: extinction probabilities in the supercritical case. Ann. Appl. Prob. 6, 922-939.

Bruss, F. T. (1984). A note on extinction criteria for bisexual Galton-Watson branching processes. J. Appl. Prob. 21, 915-919.

DALEY, D. J. (1968a). Extinction conditions for certain bisexual Galton-Watson processes. Z. Wahrscheinlichkeitsth. 9, 315-322.

Daley, D. J. (1968b). Stochastically monotone Markov chains. Z. Wahrscheinlichkeitsth. 10, 305-317.

Daley, D. J., Hull, D. A. And TaYlor, J. M. (1986). Bisexual Galton-Watson branching processes with superadditive mating functions. J. Appl. Prob. 23, 585-600.

Hull, D. M. (1982). A necessary condition for extinction in those bisexual Galton-Watson branching processes governed by superadditive mating functions. J. Appl. Prob. 19, 847-850.

Hull, D. M. (1984). Conditions for extinction in certain bisexual Galton-Watson branching processes. J. Appl. Prob. 21, 414-418.

Kamae, T., Krengel, U. and O’Brien, G. L. (1977). Stochastic inequalities on partially ordered spaces. Ann. Prob. 5, 899-912.

Klebaner, F. C. (1984). On population-size-dependent branching processes. Adv. Appl. Prob. 16, 30-45.

Molina, M., Mota, M. And Ramos, A. (2002). Bisexual Galton-Watson branching process with population-sizedependent mating. J. Appl. Prob. 39, 479-490. 